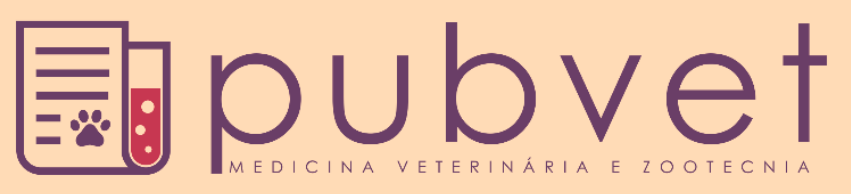

https://doi.org/10.31533/pubvet.v15n12a997.1-6

\title{
O excesso de carboidrato na dieta dos cães domésticos
}

\author{
Tarcísio Alves Barreto Filho ${ }^{1 *}$, Andressa Welison Locatel Moreira19, Beatriz De Amorim \\ Viana $^{16}$, Dhebora Crys Cunha Dos Santos ${ }^{19}$, Gabriela De Oliveira Rocha ${ }^{1}$
}

${ }^{1}$ Universidade Potiguar (UnP). Av. Sen. Salgado Filho, 1610, Lagoa Nova, Natal/RN, CEP: 59056-000;

*Autor para correspondência, E-mail: tarcisioabfilho@yahoo.com.br

\begin{abstract}
Resumo. O excesso de carboidratos em dietas voltadas à alimentação de cães pode causar o surgimento de diversas patologias, além de diminuir a qualidade de vida desses animais. Embora, pela indústria, os canídeos sejam considerados onívoros, muitos estudos científicos os classificam como carnívoros oportunistas, já que a capacidade que estes animais apresentam em digerir carboidratos não torna, necessariamente, este composto orgânico uma opção bio-apropriada para a espécie. Ao compararmos o cão e seu ancestral, o lobo cinzento, podemos encontrar diversas similaridades sobre suas preferências alimentares, entretanto, dados sobre suas necessidades nutricionais não foram amplamente estudados e debatidos. Desse modo, pode-se afirmar que a nutrição dos cães domésticos necessita de uma maior atenção.
\end{abstract}

Palavras-chave: Cão, carboidrato, exigências nutricionais, ração

\section{The excess of carbohydrates in the diet of domestic dogs}

\begin{abstract}
The excess of carbohydrates in diets aimed at feeding dogs can cause the emergence of several pathologies, in addition to decreasing the quality of life of these animals. Although, by the industry, canids are considered omnivorous, many scientific studies classify them as opportunistic carnivores, since the carbohydrates digestive capacity these animals have does not necessarily make this organic compound a bio-appropriate option for the species. When comparing the dog and its ancestor, the gray wolf, we can find several similarities about their food preferences, however, data on their nutritional needs have not been widely studied and debated. Thus, it can be said that the nutrition of domestic dogs needs greater attention.
\end{abstract}

Key words: Carbohydrate, dog, nutritional requirements, kibble

\section{Introdução}

Os carboidratos são considerados fontes de fibra e energia, e podem representar cerca de 30-60\% da matéria seca (MS) presente na dieta de cães domésticos. Esses animais são capazes de digerir parte desses macronutrientes, entretanto a composição e a estrutura dos grãos podem afetar a digestibilidade e a resposta metabólica dos cães à dieta (Carciofi, 2008).

A relação que existe entre o consumo excessivo de carboidratos e o surgimento de diversas doenças, como a obesidade foi um assunto importante apontado por Fleeman et al. (2006). A obesidade canina foi descrita como sendo associada à certos distúrbios endócrinos, como diabetes mellitus e hipotireoidismo (Daminet et al., 2003; Krook et al., 1963). A prevalência e/ou incidência de tais doenças, somada ao risco de os cães se tornarem obesos, está aumentando surpreendentemente em diferentes partes do mundo. Globalmente, foram relatados quadros de obesidade em 22,0\% a 40,0\% da população canina (Mcgreevy et al., 2005). Nos Estados Unidos da América, a prevalência variou de 34,0\% a 53,0\% (Loftus \& Wakshlag, 2015). Seja qual for a causa, a obesidade ocasiona um excesso de peso corporal 
que pode danificar os ossos e os músculos, ao exercer tensão extra nas articulações, favorecendo o surgimento de lesões em certos ligamentos (Diez \& Nguyen, 2006; Oliveira et al., 2010). Cães obesos também possuem uma certa facilidade em desenvolver alguns tipos de alterações corporais que são prejudiciais à saúde, como gordura no fígado (Joshua, 1970), redução da imunidade (Newberne, 1973), variações no metabolismo da glicose (Mattheeuws et al., 1984), problemas obstétricos (Sonnenschein et al., 1991) e desordens urinárias (Henegar et al., 2001).

Embora seja um quadro clínico comumente observado nos dias de hoje, a obesidade não deve ser tratada com descaso. Novas pesquisas devem procurar elucidar os motivos que levam o cão doméstico a apresentar uma fisionomia e uma qualidade de vida tão diferente daquela encontrada em seus ancestrais, os lobos cinzentos. É com esta ideia em mente que trazemos uma revisão literária comparativa, na qual avaliaremos as necessidades nutritivas do cão da sua cadeia evolucionaria.

\section{Origem do cão}

A domesticação de animais selvagens é um processo lento, realizado pelas adaptações fisiológicas e comportamentais de gerações previamente selecionadas e tem por finalidade aclimar certos animais ao convívio com seres humanos. Este processo implica na realização de um planejamento cauteloso e deve ser diferenciado das técnicas de adestramento e amansamento. Dentre todos, os animais já domesticados pelo ser humano, a espécie canina, foi a primeira a passar por este tipo de procedimento, há aproximadamente 14 mil anos atrás (Moody et al., 2006).

A família canidae é formada por um grupo de mamíferos carnívoros, dividido em 38 espécies (KolerMatznick, 2002). Considerando a vasta diversidade fenotípica, não se sabe ao certo quais desses animais podem ser considerados, de fato, os ancestrais do cão doméstico. Sabe-se que a biologia considera o Canis lúpus (lobo cinzento) como sendo o verdadeiro precursor do Canis lúpus familiaris (cão doméstico) devido às semelhanças encontradas na estrutura de seus DNAs mitocondriais (cerca de 98\%) (Galibert et al., 2011). Enquanto alguns estudos atuais procuram invalidar esta informação, trazendo dados sobre outros ancestrais potencialmente viáveis.

\section{Especificardes nutricionais do lobo cinzento e do cão doméstico}

Para entender os hábitos nutricionais dos cães, precisamos avaliar sua ancestralidade. Dados ecológicos sobre a alimentação de lobos selvagens indicam que esses animais são verdadeiros carnívoros, ou seja, consomem quantidades mínimas de matéria vegetal (Bosch et al., 2015). Embora essa característica esteja presente no cão doméstico, os tipos de nutrientes que esses animais consomem normalmente diferem-se daqueles observados na dieta de seus ancestrais.

A dieta do lobo selvagem é composta principalmente por animais que possuem casco (como rinocerontes, camelos, cavalos, bois e antílopes), embora também se alimentam de pequenos mamíferos, como castores, roedores e lebres (Bosch et al., 2015). A alimentação desses canídeos pode conter pequenas quantidades de material vegetal, como plantas, sementes e pequenas frutas. Alguns biólogos acreditam que a grama pode atuar como agente antiparasitário, evitando a proliferação de tênias e lombrigas, o que justifica a sua presença nas fezes de lobos selvagens. O consumo de frutas pode estar envolvido com o fato de que esses animais, diferentemente dos felinos, possuem papilas gustativas capazes de captar o sabor doce (Bradshaw, 2006).

Ao analisar a composição nutricional obtida na caça, dentre os macronutrientes, a qualidade das proteínas parece variar de acordo com o local onde elas são encontradas. As proteínas mais digestíveis estão presentes em tecidos moles, enquanto aquelas que são dificilmente digeridas encontram-se em ossos e pele (Wilmers et al., 2003). Um estudo, realizado com 50 tipos de alimentos consumidos por lobos, revelou um perfil de macronutrientes no qual 52\% da energia metabolizável parece ser adquirida da proteína, $47 \%$ da gordura e $1 \%$ de carboidratos (Bosch et al., 2015). Esse dado difere-se daquele encontrado em cães domésticos, que parecem optar por um perfil de macronutrientes em que $30 \%$ da energia metabolizável é adquirida da proteína, 63\% da gordura e 7\% dos carboidratos (Hewson-Hughes et al., 2013). Alguns autores acreditam que o aumento do consumo de lipídios ocorreu devido à domesticação desses animais no período da revolução agrícola, quando humanos passaram a oferecerlhes restos de alimentos vegetais e animais. Entretanto, é difícil acreditar que nesta época, marcada por 
momentos de penúria, pessoas abdicassem de seus alimentos para utilizá-los na dieta de cães. Logo, o mais provável é que a mudança de perfil nutritivo desses animais tenha sido ocasionada pela evolução de seus ancestrais, que buscavam consumir grandes quantidades de lipídios no intuito de aumentar suas reservas energéticas (Prentice, 2001; Prentice et al., 2005).

\section{Comparação alimentar entre o lobo selvagem e o cão doméstico}

Ao compararmos o perfil nutricional encontrado na dieta de cães domésticos e de lobos selvagens, chegaremos à conclusão de que as rações comerciais pet são completamente diferentes dos alimentos adquiridos pela caça natural (Halfen et al., 2017). A quantidade de proteína, cálcio, zinco, ferro e magnésio encontrada na dieta do Canis lupus costuma exceder o valor mínimo requerido para sobrevivência desses animais. Entretanto, na ração seca e na ração úmida, a presença de micronutrientes como $\mathrm{Ca}, \mathrm{Cu}, \mathrm{Na}$ e Mg mostra-se ainda maior (Bosch et al., 2015).

Os alimentos comerciais voltados ao mercado pet costumam apresentar, em suas composições, grandes quantidades de carboidrato. Em cães, a ingestão deste macronutriente provoca respostas metabólicas parecidas com aquelas observadas em seres humanos, nas quais a quantidade e o tipo de carboidrato atuam na variação do índice glicêmico corporal (Carciofi, 2008; Nguyen et al., 2012). Todavia, é importante compreender que, embora esses animais apresentem uma capacidade maior em digerir e absorver carboidratos pelas ações enzimáticas da amilase pancreática, o consumo repetitivo e excessivo desses compostos orgânicos pode ser prejudicial à saúde.

Os óleos (lipídios) utilizados na produção de rações, normalmente de origem animal e vegetal, apresentam uma diferença de proporção maior entre a quantidade de ômega 6 e ômega 3, podendo ser de 8:1; 5:1 e até mesmo 17:1 (Ahlstrøm et al., 2004), diferentemente do que é observado na gordura de presas selvagens. Esses compostos orgânicos são ácidos graxos essenciais, ou seja, não são sintetizados pelo organismo. Eles contribuem para prevenção de doenças, pois atuam inibindo a cascata da inflamação. Além disso, podem melhorar a condição da pelagem e da pele do animal, reduzir o aparecimento de doenças articulares e degenerativas, prevenir o aparecimento de doenças cardiovasculares, neoplasias e hipertensão, modular o sistema imunológico etc (Bosch et al., 2015). Logo, é possível concluir que em proporções erradas, fora dos parâmetros necessários do cão doméstico, a ausência ou o excesso de ômega 3 e 6 pode contribuir para o aparecimento de diversas doenças.

Embora as rações premium e super premium disponíveis no mercado apresentem quantidades aceitáveis de proteína, normalmente ela é acompanhada por teores altos de carboidratos (cerca de 30\%). $\mathrm{Na}$ alimentação ancestral, o lobo adquire toda proteína que necessita para sobreviver, sem a presença de carboidratos em excesso.

O aminoácido é a unidade estrutural da proteína, podendo ser encontrado em músculos e outros tecidos do corpo animal. Essa molécula participa ativamente em diversos processos do organismo, podendo atuar no transporte de neurotransmissores, no transporte de oxigênio, na proteção imunológica, no fornecimento de energia, na contração muscular, entre outros (Gonzalez \& Silva, 2006). Embora sejam extremamente importantes, nem todos os aminoácidos são produzidos pelo organismo, sendo necessário adquiri-los pela alimentação. Eles são comumente chamados de "aminoácidos essenciais" devido ao papel fundamental que exercem na homeostase corporal (Lehninger, 2006). Sem essas moléculas, o animal poderia vir a óbito, justamente por não conseguir nutrientes suficientes para o funcionamento de seus processos fisiológicos. Existem 20 aminoácidos considerados como padrões, e eles são responsáveis por formar todas as proteínas existentes (2005 Lehninger, 2006). Entretanto, cães e gatos só conseguem produzir metade dessas moléculas em seus organismos, sendo necessário adquirir a outra parcela pela alimentação.

Rações processadas e comidas caseiras normalmente passam por um processo de cozimento ou aquecimento, o que causa a desnaturalização de suas proteínas, danificando o fornecimento de aminoácidos. Desta forma podemos diferenciá-las da dieta bio-apropriada, pois nesta encontramos alimentos crus, nos quais os nutrientes são mantidos inalterados. 


\section{Excesso de carboidratos na dieta do cão}

Hoje, as rações secas representam a maior parte dos produtos ofertados no mercado de alimentação pet, sejam elas à base de grãos, tubérculos ou oligossacarídeos. Embora ofereçam maior praticidade na hora da utilização, esses tipos de rações apresentam uma inferioridade nutricional quando comparadas à dieta de lobos, pois são compostas de proteínas e gorduras de origem vegetal. Quando comparadas à dieta ancestral (aquelas consumidas pelo lobo cinzento), as rações apresentam uma distribuição de macronutrientes (proteínas, carboidratos e gorduras) completamente diferentes. Enquanto na caça observamos cerca de $49 \%$ de energia calórica adquirida da proteína, em rações comerciais secas esse valor chega a ser de apenas 25\% (Brown, 2009). A ração (seca ou úmida) é o alimento diário da maioria dos cães domésticos. Esse produto é submetido a processamentos que dificultam a digestão do alimento no organismo do cão, como a extrusão.

Como se trata de um procedimento termomecânico, a extrusão alimentar pode afetar as características do produto final (ração), modificando a sua digestibilidade ou a disponibilidade de nutrientes (como proteínas, carboidratos, lipídios e vitaminas). Além disso, a desnaturação de proteínas, as alterações das estruturas dos carboidratos, a oxidação dos lipídios e as reações de Maillard podem alterar a qualidade nutricional de rações extrusadas (Tran et al., 2008).

A dieta voltada aos animais de companhia contém cerca de 50\% de amido, que é derivado de diversos tipos de grãos e cereais (Buff et al., 2014; Dodd et al., 2019). Geralmente, em rações secas, as quantidades de carboidratos excedem os valores realmente necessários ao animal, enquanto a quantidade de proteína é reduzida. Este fator pode ocasionar uma série de complicações futuras no organismo canino, como: pancreatite, doenças inflamatórias intestinais, dermatopatias, endocrinopatias, entre outras. Além disso, o cão não possui amilase salivar, enzima que digere o carboidrato, sendo assim, a digestão deste macronutriente se torna mais difícil.

\section{Considerações finais}

Os dados apresentados mostram que a alimentação de lobos cinzentos, considerados os ancestrais do cão doméstico, consiste em altas quantidades de proteína e gordura, embora também apresentem os minerais, as vitaminas necessárias para o funcionamento de seus corpos.

Sobre a alimentação de cães domésticos, notamos que o teor de macronutrientes e micronutrientes encontrados em rações industrializadas, se diferem completamente daquelas encontradas nos alimentos consumidos por seus ancestrais, e este fator pode trazer malefícios a esses animais, não atendendo suas necessidades nutritivas específicas de seres carnívoros.

$\mathrm{O}$ excesso de carboidrato encontrado em diversas rações secas contraria as exigências dietéticas da espécie, visto que os cães não necessitam de carboidratos e vegetais em excesso em sua alimentação. Embora estes animais possuam uma maior capacidade em digerir e absorver esse tipo de macromolécula, isto não implica dizer que elas trazem benefícios à saúde. Essa habilidade, repassada através da evolução, foi fruto de uma adaptação para sobrevivência, não para longevidade.

Como não há muitos estudos específicos sobre as similaridades entre lobo cinzento e o cão doméstico, é de grande importância que a indústria passe a investir em pesquisas que estudem a demanda nutricional destes canídeos, a fim de melhorar a qualidade de seus produtos oferecidos no mercado.

\section{Conflito de interesse}

Declaramos que não há conflito de interesse.

\section{Referências bibliográficas}

Ahlstrøm, Ø., Krogdahl, A., Vhile, S. G., \& Skrede, A. (2004). Fatty acid composition in commercial dog foods. The Journal of Nutrition, 134(8), 2145S-2147S.

Bosch, G., Hagen-Plantinga, E. A., \& Hendriks, W. H. (2015). Dietary nutrient profiles of wild wolves: insights for optimal dog nutrition? British Journal of Nutrition, 113(S1), S40-S54.

Bradshaw, J. W. S. (2006). The evolutionary basis for the feeding behavior of domestic dogs (Canis 
familiaris) and cats (Felis catus). The Journal of Nutrition, 136(7), 1927S-1931S.

Brown, S. (2009). Unlocking the canine ancestral diet: healthier dog food the ABC way. Dogwise Publishing.

Buff, P. R., Carter, R. A., Bauer, J. E., \& Kersey, J. H. (2014). Natural pet food: A review of natural diets and their impact on canine and feline physiology. Journal of Animal Science, 92(9), 3781-3791. https://doi.org/10.2527/jas.2014-7789.

Carciofi, A. C. (2008). Fontes de proteína e carboidratos para cães e gatos. Revista Brasileira de Zootecnia, 37(SPE), 28-41. https://doi.org/10.1590/S1516-35982008001300005.

Daminet, S., Jeusette, I., Duchateau, L., Diez, M., Van de Maele, I., \& De Rick, A. (2003). Evaluation of thyroid function in obese dogs and in dogs undergoing a weight loss protocol. Journal of Veterinary Medicine Series A, 50(4), 213-218. https://doi.org/10.1046/j.1439-0442.2003.00534.x.

Diez, M., \& Nguyen, P. (2006). Obesity: epidemiology, pathophysiology and management of the obese dog. Encyclopedia of Canine Clinical Nutrition, 2-53.

Dodd, S. A. S., Cave, N. J., Adolphe, J. L., Shoveller, A. K., \& Verbrugghe, A. (2019). Plant-based (vegan) diets for pets: A survey of pet owner attitudes and feeding practices. PloS One, 14(1), e0210806. https://doi.org/10.1371/journal.pone.0210806.

Fleeman, L. M., Seton, E. J., \& Rand, J. (2006). Como abordar: O manejo da obesidade em cães e gatos. Veterinary Focus, 16, 9-15.

Galibert, F., Quignon, P., Hitte, C., \& André, C. (2011). Toward understanding dog evolutionary and domestication history. Comptes Rendus Biologies, 334(3), 190-196.

Gonzalez, F. H. D., \& Silva, S. C. (2006). Introdução à bioquímica clínica animal. Gráfica de Universidade Federal do Rio Grande do Sul.

Halfen, D. P., Oba, P. M., Duarte, C. N., Santos, J. P. F., Vendramini, T. H. A., Sucupira, M. C. A., Carciofi, A. C., \& Brunetto, M. (2017). Tutores de cães consideram a dieta caseira como adequada, mas alteram as fórmulas prescritas. Pesquisa Veterinária Brasileira, 37(12), 1453-1459.

Henegar, J. R., Bigler, S. A., Henegar, L. K., Tyagi, S. C., \& Hall, J. E. (2001). Functional and structural changes in the kidney in the early stages of obesity. Journal of the American Society of Nephrology, 12(6), 1211-1217.

Hewson-Hughes, A. K., Hewson-Hughes, V. L., Colyer, A., Miller, A. T., McGrane, S. J., Hall, S. R., Butterwick, R. F., Simpson, S. J., \& Raubenheimer, D. (2013). Geometric analysis of macronutrient selection in breeds of the domestic dog, Canis lupus familiaris. Behavioral Ecology, 24(1), 293-304.

Joshua, J. O. (1970). The obese dog and some clinical repercussions. Journal of Small Animal Practice, 11(9), 601-606. https://doi.org/10.1111/j.1748-5827.1970.tb05621.x.

Koler-Matznick, J. (2002). The origin of the dog revisited. Anthrozoös, 15(2), 98-118.

Krook, L., Barrett, R. B., Usui, K., \& Wolke, R. E. (1963). Nutritional secondary hyperparathyroidism in the cat. Cornell Veterinarian, 53, 224-240.

Lehninger, N. D. L. (2006). Principios de bioquímica. São Paulo.

Loftus, J. P., \& Wakshlag, J. J. (2015). Canine and feline obesity: a review of pathophysiology, epidemiology, and clinical management. Veterinary Medicine: Research and Reports, 6, 49. https://doi.org/10.2147/vmrr.s40868.

Mattheeuws, D., Rottiers, R., Kaneko, J. J., \& Vermeulen, A. (1984). Diabetes mellitus in dogs: relationship of obesity to glucose tolerance and insulin response. American Journal of Veterinary Research, 45(1), 98-103.

Mcgreevy, P. D., Thomson, P. C., Preide, C., Fawcett, A., Grassi, T. \& Jones, B. (2005). Prevalence of obesity in dogs examined by Australian veterinary practices and the risk factors involved. Veterinary Record, 156(22), 695-702,

Moody, J. A., Clark, L. A., \& Murphy, K. E. (2006). Working dog: History and applications. In E. A. Ostrander, U. Giger, \& K. Lindblad-Toh (Eds.), The dog and its genome. Cold Spring Harbor Laboratory Press. 
Newberne, P. M. (1973). influence of nutrition response to infectious disease. Advances in Veterinary Science and Comparative Medicine, 17, 265-289.

Nguyen, N. Q., Besanko, L. K., Burgstad, C., Bellon, M., Holloway, R. H., Chapman, M., Horowitz, M., \& Fraser, R. J. L. (2012). Delayed enteral feeding impairs intestinal carbohydrate absorption in critically ill patients. Critical Care Medicine, 40(1), 50-54.

Oliveira, M. C., Nascimento, B. C. L., \& Amaral, R. W. C. (2010). Obesidade em cães e seus efeitos em biomarcadores sanguíneos-revisão de literatura. PUBVET, 4, Art. 795-801.

Prentice, A. M. (2001). Fires of life: the struggles of an ancient metabolism in a modern world. Nutrition Bulletin, 26(1), 13-27.

Prentice, A. M., Rayco-Solon, P., \& Moore, S. E. (2005). Insights from the developing world: thrifty genotypes and thrifty phenotypes. Proceedings of the Nutrition Society, 64(2), 153-161.

Sonnenschein, E. G., Glickman, L. T., Goldschmidt, M. H., \& McKee, L. J. (1991). Body conformation, diet, and risk of breast cancer in pet dogs: a case-control study. American Journal of Epidemiology, 133(7), 694-703. https://doi.org/10.1093/oxfordjournals.aje.a115944.

Tran, Q. D., Hendriks, W. H., \& van der Poel, A. F. B. (2008). Effects of extrusion processing on nutrients in dry pet food. Journal of the Science of Food and Agriculture, 88(9), 1487-1493.

Wilmers, C. C., Crabtree, R. L., Smith, D. W., Murphy, K. M., \& Getz, W. M. (2003). Trophic facilitation by introduced top predators: grey wolf subsidies to scavengers in Yellowstone National Park. Journal of Animal Ecology, 72(6), 909-916.

Histórico do artigo:

Recebido: 28 de julho de 2021

Aprovado: 15 de setembro de 2021

Disponível on-line: 25 de novembrode 2021
Licenciamento: Este artigo é publicado na modalidade Acesso Aberto sob a licença Creative Commons Atribuição 4.0 (CC-BY 4.0), a qual permite uso irrestrito, distribuição, reprodução em qualquer meio, desde que o autor e a fonte sejam devidamente creditados. 